\title{
Potential alternative treatment approach for pediatric patient with diffusely infiltrative primary rhabdomyosarcoma of the liver
}

\author{
Rushil Patel ${ }^{1 \star}$, John Roberson ${ }^{2 \star}$, Devina Prakash ${ }^{3}$, Rina Meyer ${ }^{3}$, Laura Hogan ${ }^{3}$, Christeen Azmy ${ }^{3}$, \\ Valmore Suprenant ${ }^{4}$, Samuel Ryu ${ }^{2}$, Alexander Stessin ${ }^{2}$ \\ ${ }^{1}$ Renaissance School of Medicine, Stony Brook University, Stony Brook, NY, United States \\ ${ }^{2}$ Department of Radiation Oncology, Stony Brook University Hospital, Stony Brook, NY, United States \\ ${ }^{3}$ Division of Pediatric Hematology Oncology, Stony Brook Children's Hospital, Stony Brook, NY, United States \\ ${ }^{4}$ Department of Radiology, Stony Brook University Hospital, Stony Brook, NY, United States \\ ${ }^{*}$ Co-first authors
}

\begin{abstract}
Primary hepatic rhabdomyosarcoma is rare, making decisions regarding locoregional management with resection and/or conventional radiation difficult. We present a novel treatment approach for a pediatric patient diagnosed with rhabdomyosarcoma diffusely involving the liver. This patient underwent treatment with yttrium-90 (Y-90) microspheres followed by external beam radiation therapy (EBRT) to residual disease, interdigitated with systemic chemotherapy. Initial post-radiation imaging showed significant response to treatment, and she experienced minimal acute toxicities and no long-term toxicities. She developed recurrent PET-avid disease 23 months after $Y-90$ treatment, necessitating further local and continued systemic therapies. We report on the tumor control following Y-90 and EBRT treatment.
\end{abstract}

Key words: yttrium-90; external beam radiation therapy; pediatrics; rhabdomyosarcoma

Rep Pract Oncol Radiother 2021;26(1):143-148

\section{Introduction}

Rhabdomyosarcoma is a malignant soft tissue cancer that commonly manifests in the skeletal muscle, accounting for approximately $3 \%$ of malignant tumors in children and associated with variable overall survival based on risk stratification [1, 2]. Typical management includes chemotherapy and surgical resection frequently followed by radiation therapy; however, resection may not be feasible in select cases [3]. One such location can be the liver. Although external beam radiation therapy (EBRT) is typically employed in unresectable cases, diffuse liver involvement presents a problem due to the risk of irreversible and even fatal radiationinduced liver disease. Such cases, therefore, necessitate the use of other local treatment strategies.

Selective internal radiation therapy (SIRT) with yttrium-90 (Y-90) is a minimally invasive procedure that takes advantage of the liver's dual blood supply, in which hepatocytes are primarily supplied by the portal vein and tumor cells by the hepatic artery, to preferentially deliver beta-emitting Y-90 microspheres to tumors [4]. This allows delivery of higher radiation doses to tumors while selectively sparing healthy hepatocytes and is frequently utilized to treat both primary and metastatic liver tumors in adults $[5,6]$. SIR-Spheres, one type of Y-90 microsphere,

Address for correspondence: Alexander Stessin MD, PhD, Stony Brook University, Stony Brook, NY 11794, United States, tel: 631-444-221; e-mail: Alexander.Stessin@stonybrookmedicine.edu

This article is available in open access under Creative Common Attribution-Non-Commercial-No Derivatives 4.0 International (CC BY-NC-ND 4.0) license, allowing to download articles and share them with others as long as they credit the authors and the publisher, but without permission to change them in any way or use them commercially 
have been designed to be small enough to access tumor microvasculature but large enough to remain within the capillary bed to limit toxic effects [7].

Y-90 is generally well-tolerated with minimal side effects in adults, often limited to mild fatigue and abdominal discomfort [8]. However, there are very few reports of its tolerability and efficacy in pediatric patients. A case series published in 2018 of 10 children and adolescents reported 4 patients developing fatigue and fevers without signs of infection, suggesting it may be safe in pediatric patients [9].

Here, we present the case of a pediatric patient with rhabdomyosarcoma with diffuse liver involvement treated at our institution with bilobar SIR-Spheres followed by EBRT to residual disease. We present the safety and efficacy of her treatment course and its implications for Y-90 treatment in pediatric patients with diffuse liver involvement. We also delineate dosimetric considerations when combining Y-90 and EBRT, a treatment approach which may be considered for similar cases in the future.
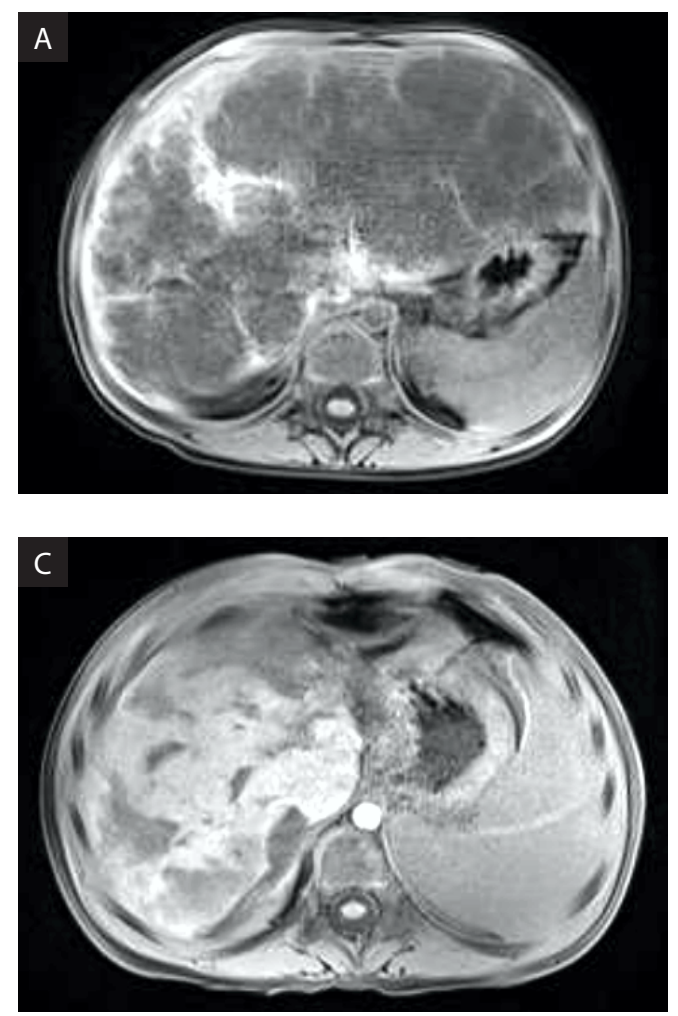

\section{Case report}

A 5-year-old female with no significant past medical history presented with 2 days of abdominal pain and anorexia and a palpably enlarged liver. MRI showed a large heterogeneous liver with innumerable confluent multiseptated lesions compatible with diffuse involvement, portal vein thrombosis, and grade 1 esophageal and gastric varices (Fig. 1A). Ultrasound-guided biopsy of the liver mass demonstrated embryonal rhabdomyosarcoma, and PET/MRI showed disease diffusely involving the liver with potential tumor thrombus in the portal and superior mesenteric veins. She was started on induction chemotherapy with vincristine, dactinomycin, and cyclophosphamide alternating with vincristine and irinotecan/topotecan for 9 months, during which she developed expected side effects including fever, vomiting, weight loss, and abdominal pain. Follow-up MRI showed persistent hepatomegaly with slight decrease in the
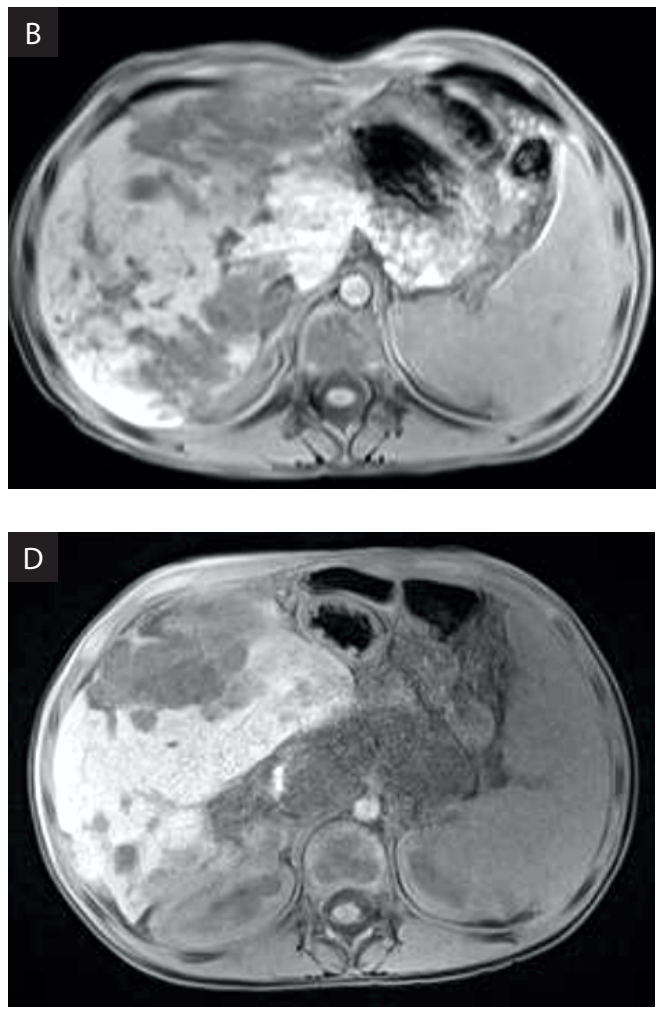

Figure 1. Axial abdominal MRI images throughout treatment course. A. MRI on day of diagnosis showing heterogeneous hepatomegaly compatible with diffuse tumor involvement; portal vein thrombosis also present. B. MRI after initial chemotherapy regimen demonstrating persistent hepatomegaly with mild improvement of intrahepatic disease burden; residual mild enhancement in portal vein, proximal mesenteric vein, and splenic vein. C. MRI 14 weeks after Y-90 treatment showing mixed response of intrahepatic tumor and extensive tumor growth within the portal venous system with distension of superior mesenteric vein and splenic vein. D. MRI prior to salvage EBRT showing mixed response to salvage chemotherapy with areas of tumor progression in liver segments 5 and 8 and the splenoportal system 


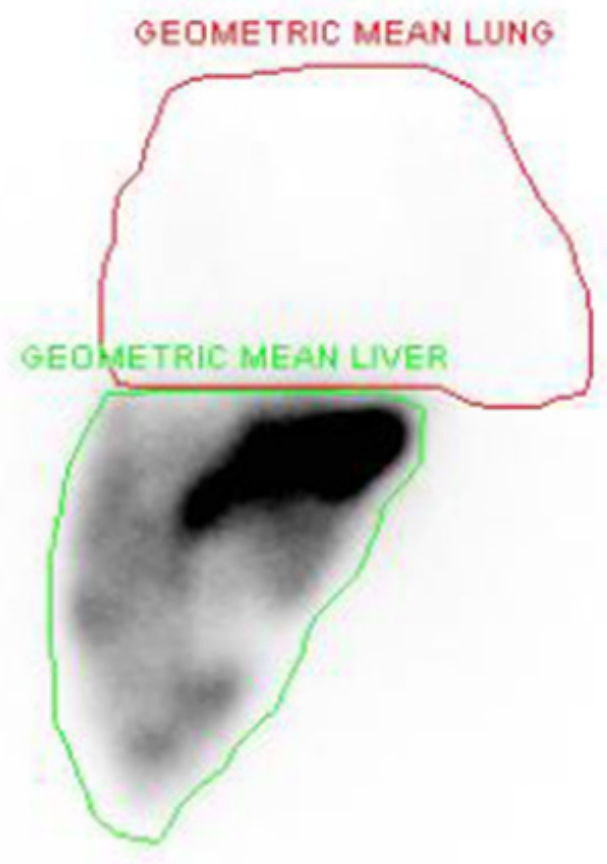

Figure 2. Yttrium-90 mapping angiogram demonstrating extensive tumor involvement throughout both hepatic lobes with lung shunt $6.2 \%$

extent of intrahepatic disease (e.g., conglomerate right lobe lesions measured $5.6 \times 5.3 \mathrm{~cm}$ from 6.5 $\times 6.7 \mathrm{~cm}$ ) and a mild enhancement of surrounding veins (Fig. 1B).

However, due to persistent diffuse liver involvement, neither surgical resection nor EBRT was considered feasible, and Y-90 microspheres were suggested as a non-standard treatment option. Mapping angiogram (Fig. 2) demonstrated extensive tumor throughout both hepatic lobes. The lung shunt was acceptable at $6.2 \%$ (radiation pneumonitis may be seen with lung shunts $>20 \%$ ). Protective coil embolism of an accessory splenic artery arising from the gastrohepatic trunk was performed to prevent SIR-Spheres flowing through this vessel and potentially damaging healthy tissue. The body surface area method was used to calculate the dose of SIR-Spheres [10]. She initially received $18.8 \mathrm{mCi}$ to the right lobe without complication. Four weeks later, she received $3.9 \mathrm{mCi}$ to the left lobe, at which point stasis was noted as there was no antegrade blood flow seen on angiography preventing delivery of the entire calculated activity of microspheres. MRI 14 weeks later showed mixed response within the liver with extensive tumor growth in unirradiat-

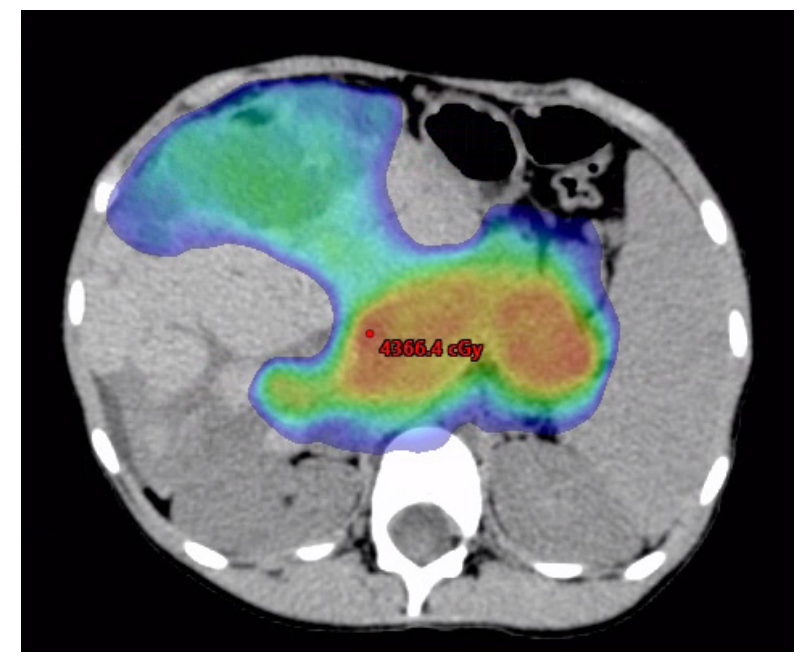

Figure 3. External beam radiation therapy plan showing intrahepatic volume treated with $30 \mathrm{~Gy}$ in 10 fractions and extrahepatic volume treated to $42 \mathrm{~Gy}$ in 14 fractions

ed portal venous branches causing distension of the superior mesenteric vein and splenic vein (Fig. 1C).

She began salvage chemotherapy with vincristine, cyclophosphamide, and temsirolimus; however, due to poor tolerability, this regimen was discontinued. Follow-up MRI 8 weeks after initiating salvage chemotherapy showed areas of decreased tumor bulk, likely reflecting treatment response, but multiple other areas of tumor progression, particularly within segments 5 and 8 and the splenoportal system (Fig. 1D).

Due to this progression, she was considered for EBRT which had been reported to be safe after Y-90 in adults [11]. Decision was made to proceed with definitive EBRT to all known disease, including 42 Gy in 14 fractions to the extrahepatic disease and a dose-reduction to $30 \mathrm{~Gy}$ in 10 fractions to the intrahepatic tumor due to the prior Y-90 treatment (Fig. 3). Table 1 includes dose constraints used for this EBRT treatment, which was well tolerated with only mild abdominal pain. Review of liver function tests (LFTs) showed no grade 2 or higher adverse events according to Common Terminology Criteria for Adverse Events version 5 after either radiation course [12]. Please see Table 2 for the evolution of the LFTs from diagnosis to post-EBRT.

After completing EBRT, she continued chemotherapy with vincristine, epirubicin, and carboplatin alternating with ifosfamide, etoposide, and vincristine for a total of 9 cycles over 11 months, during which she required multiple prolonged hos- 
Table 1. Dose constraints used for external beam radiation therapy (EBRT) treatment. Liver dose constraints adopted from [11]. Kidney and spinal cord constraints were department standard dose constraints, as adopted from QUANTEC [19]

\begin{tabular}{|l|ll|}
\hline \multirow{4}{*}{ Organs at risk } & \multicolumn{2}{l|}{ Dose (Gy) or dose/volume parameters } \\
\hline \multirow{4}{*}{ Liver } & Mean dose & $\leq 2000$ cGy \\
\cline { 2 - 3 } & V500 cGy & $\leq 86 \%$ \\
\cline { 2 - 3 } & V1000 cGy & $\leq 68 \%$ \\
\cline { 2 - 3 } & V2000 cGy & $\leq 49 \%$ \\
\cline { 2 - 3 } & V3000 cGy & $\leq 28 \%$ \\
\cline { 2 - 3 } & V4000 cGy & $\leq 20 \%$ \\
\hline Kidneys (each) & Mean dose & $\leq 1800$ cGy \\
\hline & V2000 cGy & $\leq 32 \%$ \\
\hline Spinal cord & Max dose & $\leq 4500$ cGy \\
\hline
\end{tabular}

pitalizations (e.g., sepsis, rectal bleeding, and Clostridium difficile infection). Follow-up PET/CT 6 months after EBRT showed complete resolution of PET activity with persistent non-avid mass in portal, splenic, and superior mesenteric veins. However, on imaging 15 months after salvage EBRT, she was found to have recurrence within the irradiated liver and portal vein and marginally irradiated retroperitoneum. Due to prior Y-90 and EBRT to the liver, she underwent microwave ablation of the liver lesion with transient elevation of liver enzymes. She also received stereotactic body radiation therapy to the portal vein and retroperitoneal recurrences to $25 \mathrm{~Gy}$ in 5 fractions without acute or subacute toxicity. She subsequently developed further progression intrahepatically and in the portal vein, requiring biliary stent placement. She ultimately died from ascending cholangitis from multi-drug resistant Enterobacter cloacae 29 months after her Y-90 treatment.

\section{Discussion}

Local therapy for rhabdomyosarcoma is primarily dictated by tumor location and underlying histology. However, in patients with diffuse liver involvement, both surgery and EBRT may be unfeasible, necessitating the use of novel therapies.

Treatment with Y-90 can be used to safely deliver higher radiation doses to liver tumors than EBRT [13]. While studies have shown improved survival rates in adults treated with Y-90 [14-16], such studies in pediatric patients are very few. Hawkins et al. (2013) reported two children with hepatocellular carcinoma treated with Y-90 who survived 4 months and 20 months post-treatment [17]. Aguado et al. (2018) reported the treatment of 10 pediatric patients with primary hepatic malignancies with Y-90 with survival ranging from 2-20 months and 3 patients tolerating re-treatment with Y-90 [9]. Our patient survived 26 months following Y-90 treatment (and 41 months after diagnosis) with minimal toxicity from her local therapies, despite further EBRT and microwave ablation to the liver.

The selection of local therapy for rhabdomyosarcoma involving the liver is further complicated by the known potential hepatotoxicity of several of the agents used in the treatment of rhabdomyosarcoma [18]. For example, veno-occlusive disease is considered a common side effect of cyclophosphamide,

Table 2. Evolution of liver function tests from time of diagnosis to post-EBRT treatment

\begin{tabular}{|c|c|c|c|c|c|}
\hline Measurement & Diagnosis & Prior to Y-90 & After Y-90 & Prior to EBRT & After EBRT \\
\hline INR & 1.3 & 1.2 & 1.3 & - & - \\
\hline PTT & 35.4 & $59.5^{*}$ & 44.4 & - & - \\
\hline Bilirubin, total & 1.7 & 0.4 & 0.4 & 0.7 & 1.3 \\
\hline Bilirubin, direct & 1.1 & $<0.2$ & $<0.2$ & 0.3 & 0.6 \\
\hline ALT & 144 & 56 & 60 & 81 & 102 \\
\hline AST & 145 & 49 & 70 & 82 & 68 \\
\hline ALP & 527 & 158 & - & - & 360 \\
\hline Protein, total & 5.9 & 7.2 & - & - & 5.9 \\
\hline Albumin & 3.3 & 4.6 & - & - & 3.3 \\
\hline
\end{tabular}

*indicates grade $\geq 2$ severity as per CTCAE version 5.0; EBRT — external beam radiation therapy; INR — international normalized ratio (normal range: 0.8-1.2); PTT — partial thromboplastin time in seconds (normal range: 22.2-36.0); total bilirubin in $\mathrm{mg} / \mathrm{dL}$ (normal range: $0.0-1.2$ ); direct bilirubin in mg/dL (normal range: 0.0-0.3); ALT — alanine aminotransferase in IU/L (normal range: 0-33); AST — aspartate aminotransferase in IU/L (normal range: 0-32); ALP — alkaline phosphatase in IU/L (normal range: $39-390$ ); total protein in $\mathrm{g} / \mathrm{dL}$ (normal range: 6.4-8.3); albumin in g/dL (normal range: $3.5-4.8$ ) 
resulting in toxicity in $38 \%$ of patients who receive it in conjunction with total body irradiation. Similarly, other forms of hepatotoxicity including hepatitis, cholestasis, and steatosis are known to occur following administration of vincristine, actinomycin-D, irinotecan, topotecan, ifosfamide, and etoposide all of which she received at some point during the treatment of her rhabdomyosarcoma. Further, some of these side effects may be potentiated by radiation therapy. Despite this, she only experienced a grade 2 prolongation of her PTT from her chemotherapy regimens and did not demonstrate any other grade 2 or higher side effects following administration of Y-90, EBRT, or salvage chemotherapy regimens.

Here, we present a case of rhabdomyosarcoma diffusely involving the liver where no standard therapies were feasible. Y-90 followed by EBRT was well tolerated with minimal toxicity in our case. As rhabdomyosarcoma diffusely involving the liver is rare, trials establishing a standard of care for such patients are not feasible. However, prospective studies evaluating the safety and efficacy of Y-90 alone or followed by EBRT to residual disease in children with unresectable liver tumors may be possible. Treatment with Y-90 followed by salvage EBRT, as demonstrated in this case report, can be delivered both safely and effectively in children.

\section{Conflict of interest}

The authors declare that they have no conflict of interest related to this manuscript.

\section{Funding}

No funding was received for the preparation of this manuscript.

\section{Authors' contribution}

I - conception and design: A.S., V.S., S.R, L.H., D.P., R.M.; II - administrative support: S.R., A.S., D.P., L.H., R.M., C.A.; III - provision of study materials or patients: none; IV - collection and assembly of data: R.P., R.R.; V - data analysis and interpretation: none; VI - manuscript writing: R.P., J.R.; VII - final approval of manuscript: all authors.

\section{References}

1. Howlader N, Noone AM, Krapcho M, Miller D, Brest A, Yu M, et al. SEER Cancer Statistics Review, 1975-2017, National Cancer Institute. Bethesda, MD. https://seer.cancer.gov/ csr/1975_2017/ (September 10, 2020).
2. Gartrell J, Pappo A. Recent advances in understanding and managing pediatric rhabdomyosarcoma. F1000Res. 2020; 9, doi: 10.12688/f1000research.22451.1, indexed in Pubmed: 32695311.

3. Crist W, Gehan EA, Ragab AH, et al. The Third Intergroup Rhabdomyosarcoma Study. J Clin Oncol. 1995; 13(3): 610-630, doi: 10.1200/JCO.1995.13.3.610, indexed in Pubmed: 7884423.

4. Bierman HR, Byron RL, Kelley KH, et al. Studies on the blood supply of tumors in man. III. Vascular patterns of the liver by hepatic arteriography in vivo. J Natl Cancer Inst. 1951; 12(1): 107-131, indexed in Pubmed: 14874125.

5. Wang EA, Broadwell SR, Bellavia RJ, et al. Selective internal radiation therapy with SIR-Spheres in hepatocellular carcinoma and cholangiocarcinoma. J Gastrointest Oncol. 2017; 8(2): 266-278, doi: 10.21037/jgo.2016.11.08, indexed in Pubmed: 28480066.

6. Llovet JM, Ricci S, Mazzaferro V, et al. SHARP Investigators Study Group. Sorafenib in advanced hepatocellular carcinoma. N Engl J Med. 2008; 359(4): 378-390, doi: 10.1056/ NEJMoa0708857, indexed in Pubmed: 18650514.

7. Mosconi C, Cappelli A, Pettinato C, et al. Radioembolization with Yttrium-90 microspheres in hepatocellular carcinoma: Role and perspectives. World J Hepatol. 2015; 7(5): 738-752, doi: 10.4254/wjh.v7.i5.738, indexed in Pubmed: 25914774.

8. Riaz A, Awais R, Salem R. Side effects of yttrium-90 radioembolization. Front Oncol. 2014; 4: 198, doi: 10.3389/ fonc.2014.00198, indexed in Pubmed: 25120955.

9. Aguado A, Ristagno R, Towbin AJ, et al. Transarterial radioembolization with yttrium-90 of unresectable primary hepatic malignancy in children. Pediatr Blood Cancer. 2019; 66(7): e27510, doi: 10.1002/pbc.27510, indexed in Pubmed: 30406959.

10. Kennedy AS, McNeillie P, Dezarn WA, et al. Treatment parameters and outcome in 680 treatments of internal radiation with resin $90 \mathrm{Y}$-microspheres for unresectable hepatic tumors. Int J Radiat Oncol Biol Phys. 2009; 74(5): 1494-1500, doi: 10.1016/j.jijrobp.2008.10.005, indexed in Pubmed: 19157721.

11. Wang TH, Huang PI, Hu YW, et al. Combined Yttrium-90 microsphere selective internal radiation therapy and external beam radiotherapy in patients with hepatocellular carcinoma: From clinical aspects to dosimetry. PLoS One. 2018; 13(1): e0190098, doi: 10.1371/journal. pone.0190098, indexed in Pubmed: 29293557.

12. Common terminology criteria for adverse events (CTCAE) v5. https://ctep.cancer.gov/protocoldevelopment/electronic_applications/docs/CTCAE_v5_Quick_ Reference_5x7.pdf. (June 2, 2020).

13. Lewandowski RJ, Geschwind JF, Liapi E, et al. Transcatheter intraarterial therapies: rationale and overview. Radiology. 2011; 259(3): 641-657, doi: 10.1148/radiol.11081489, indexed in Pubmed: 21602502.

14. D'Avola D, Lñarrairaegui M, Bilbao Jl, et al. A retrospective comparative analysis of the effect of Y90-radioembolization on the survival of patients with unresectable hepatocellular carcinoma. Hepatogastroenterology. 2009; 56(96): 1683-1688, indexed in Pubmed: 20214218.

15. Lewandowski RJ, Kulik LM, Riaz A, et al. A comparative analysis of transarterial downstaging for hepatocelIular carcinoma: chemoembolization versus radioembolization. Am J Transplant. 2009; 9(8): 1920-1928, 
doi: 10.1111/j.1600-6143.2009.02695.x, indexed in Pubmed: 19552767.

16. Salem R, Lewandowski RJ, Mulcahy MF, et al. Radioembolization for hepatocellular carcinoma using Yttrium-90 microspheres: a comprehensive report of long-term outcomes. Gastroenterology. 2010; 138(1): 52-64, doi: 10.1053/j.gastro.2009.09.006, indexed in Pubmed: 19766639.

17. Hawkins CM, Kukreja K, Geller Jl, et al. Radioembolisation for treatment of pediatric hepatocellular carcinoma.
Pediatr Radiol. 2013; 43(7): 876-881, doi: 10.1007/s00247012-2568-y, indexed in Pubmed: 23212597.

18. Grigorian A, O'Brien CB. Hepatotoxicity Secondary to Chemotherapy. J Clin Transl Hepatol. 2014; 2(2): 95-102, doi: 10.14218/JCTH.2014.00011, indexed in Pubmed: 26357620.

19. Marks LB, Yorke ED, Jackson A, et al. Use of normal tissue complication probability models in the clinic. Int J Radiat Oncol Biol Phys. 2010; 76(3 Suppl): S10-S19, doi: 10.1016/j. ijrobp.2009.07.1754, indexed in Pubmed: 20171502. 